\title{
Combining Appearance and Range Based Information for Multi-class Generic Object Recognition
}

\author{
Doaa Hegazy and Joachim Denzler \\ Institute of Computer Science, Friedrich-Schiller-University in Jena, \\ Ernst-Abbe-Platz 2, 07743 Jena, Germany \\ doaa.hegazy@uni-jena.de, joachim.denzler@uni-jena.de
}

\begin{abstract}
The use of range images for generic object recognition is not addressed frequently by the computer vision community. This paper presents two main contributions. First, a new object category dataset of $2 \mathrm{D}$ and range images of different object classes is presented. Second, a new generic object recognition model from range and $2 \mathrm{D}$ images is proposed. The model is able to use either appearance (2D) or range based information or a combination of both of them for multi-class object learning and recognition. The recognition performance of the proposed recognition model is investigated experimentally using the new database and promising results are obtained. Moreover, the best performance gain by combining both appearance and range based information is $35 \%$ for single classes while the average gain over classes is $12 \%$.
\end{abstract}

\section{Introduction}

Generic object recognition (GOR) has been an important topic of the computer vision research in recent years. Many different approaches have been developed to give a solution to such difficult problem (e.g. [2, 7]). However, most of the successful approaches developed up to date have concentrated on generic recognition of objects from 2D images, and very little attention has been paid to the use of $3 \mathrm{D}$ range data. Range images have the advantage of providing direct cues of the shape of objects which is important for representing and recognizing different visual object classes.

However, the absence of GOR work using range images is due to two main reasons: 1) the non-availability of an object category dataset which provides range data (images) of its member classes. The currently available object category datasets which emerged as standards for the GOR community provide only 2D images of their object categories such as Caltech-6, Caltech 101 [1] and Graz [7, 2) surface shape representation is very important in a recognition procedure from range data in general. However, it is not clear which representation is more suitable for learning shapes of visual object classes. Authors in [8] have developed an approach to recognize objects belonging to a particular shape class in range images and presented a shape representation called symbolic surface signature. The dataset used for learning and classifying their model is a set of range

E. Bayro-Corrochano and J.-O. Eklundh (Eds.): CIARP 2009, LNCS 5856, pp. 741-748, 2009.

(C) Springer-Verlag Berlin Heidelberg 2009 
images of objects made of clay. Many important differences exist between the model proposed here and the one in [8]. Among these differences is that in our approach, combination of appearance (2D) and range based information is used for GOR recognition which is not the case in 8].

This paper addresses the use of range images for GOR and presents two main contributions. First, a new object category dataset is constructed. The dataset provides 2D (color) as well as $3 \mathrm{D}$ range images of its member classes, with dense background clutter and occlusion. The availability of the two different image types makes the dataset suitable for GOR from either $2 \mathrm{D}$ or range data or from a combination of both data types. Moreover, it can be used for both $2 \mathrm{D}$ and 3D GOR as well. The second contribution is a new model for GOR with the following advantages: 1) it recognizes generic object classes from range images by exploiting shape cues of objects, 2) it is based on local representation of range images by using interest regions detection and description. Therefore, the model is able to recognize objects in range images despite background clutter and occlusion, 3) performs multi-class learning and recognition of generic object classes from range images, 4) the general framework of the recognition model allows the use of $2 \mathrm{D}$ images as well for recognition using either texture or color information or both of them and 5) the framework gives the ability to combine both appearance (2D) and shape (range) cues for GOR of multiple classes.

The outline of the remainder of this paper is as follows. Section 2 is devoted to describe the new object category dataset. The proposed generic object recognition model is described in section 3. Experimental evaluations of the proposed model as well as results obtained are presented in section 4 . Conclusions are finally drawn in section 5 .

\section{An Object Category Dataset}

An object category dataset of 4220 2D/3D images (2D colored and range images) of 35 objects was constructed using a 3D Time-of-Flight (TOF) PMD camera 4 ] and a CCD camera. The objects are instances of five visual classes (categories): cars, toys, cups, fruits and animals (see figure 1 (a)). For each object category, seven individual instances were used. Due to the difficulty to record different outdoor views of natural objects using the TOF camera, indoor views in an office environment were captured. Artificial objects were used in replacement of real instances of some visual classes (namely cars and animals) in building the dataset. The instances of each object class were chosen with different sizes and appearance to achieve large intra-class variabilities as much as possible (see figure 1(a)). Many images of the dataset contain multiple instances of the same class or from different classes. Moreover, the images contain large viewpoint and orientation variations, partial occlusion (e.g. by other objects) and truncation (e.g. by the image boundaries) as well as background clutter (see figure 1 (c)). The images of each individual object instance were acquired under eight different viewing angles and four different heights. This is accomplished as follows: at each height, each object instance was placed on a turn table which was rotated 


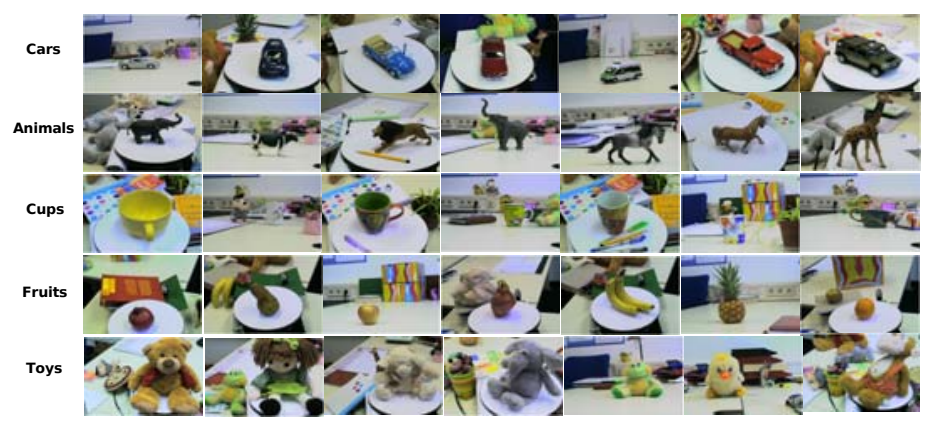

(a)

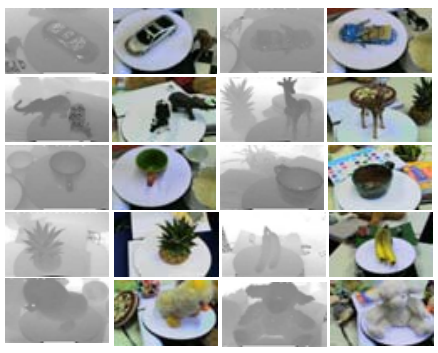

(b)

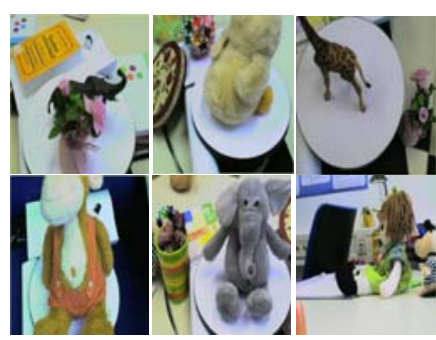

(c)

Fig. 1. (a) Example images of the five object classes of the new object category dataset. (b) Example range range images (using TOF camera) and their corresponding color images (using CCD camera). TOF cameras produce reflected images with respect to images produced by CCD cameras. (c) Example images of the dataset with occlusion and truncation.

through 360 degrees about the vertical axis and eight colored and range images were acquired; one at every 45 degrees. The total number of images acquired using each camera is 32 images for each object instance ( 4 heights $\times 8$ angles). The TOF camera delivers also an intensity image corresponding to each range image. However, the delivered intensity image is of low resolution which affects direct application of some image processing algorithms on them.

The dataset will be available for public use 1 as we believe that the dissemination and use of this dataset will allow realistic comparative studies as well as a source to test data for development for new techniques of GOR from range images.

\section{The Recognition Model}

Figure 2 displays a semantic view of the general framework of the proposed GOR model. As shown in the figure, the recognition model consists of three main steps, which are described in this section.

\footnotetext{
${ }^{1}$ http://www.inf-cv.uni-jena.de/index.php?id=dataset
} 


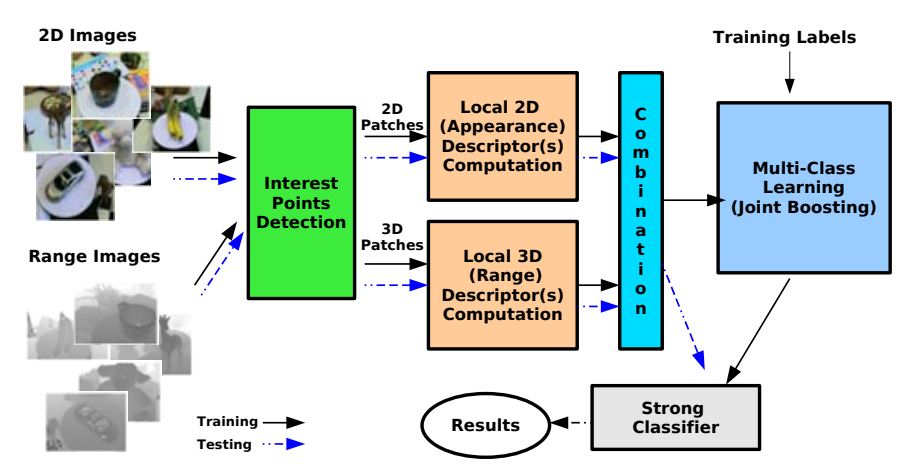

Fig. 2. The general framework of the proposed GOR model

\subsection{Interest Points Detection}

An interest point detector, namely the Hessian affine-invariant point detector 6], is run on 2D images to detect a set of interest points. For the range images, the interest point detector is run on the intensity images corresponding to range images delivered by the TOF camera. Afterwards, the $3 \mathrm{D}$ regions corresponding to the detected points are extraced. However, the range data of a TOF camera suffer from large amount of noise. In order to filter some of this noise and smooth the range data, a preprocessing step by applying a median filter is first performed. Furthermore, an initial histogram normalization is applied to the TOF intensity images to enhance their low contrast before interest points detection.

\subsection{Local Description}

Based on the type of the extracted region (2D or $3 \mathrm{D})$, a local descriptor is computed from it. A set of different local descriptors is used for both types of data including grayscale, color and shape descriptors.

2D Descriptors. Two different types of descriptors are used: the SIFT descriptors [5] and the opponent color angle descriptors [10].

3D (Range) Descriptors. Three shape-specific local feature histograms are used. These features were presented and used in 3 for the task of free-form specific 3D object recognition. The features are namely: pixel depth, surface normals and curvature. The main advantages of these features are that they are easy to calculate, robust to viewpoint changes and contain discriminative information [3]. For the lack of available space, more information about the features are found in 3 . 

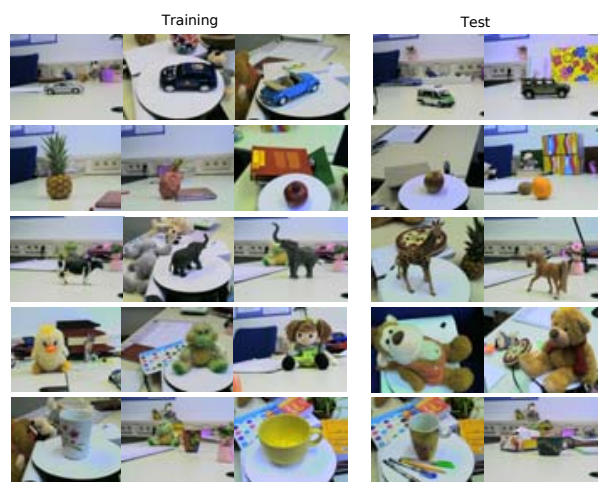

Fig. 3. Object class instances used to train and test the proposed model

\subsection{Learning Model}

Learning in our model is based on the Joint Boosting algorithm [9] which depends on training multiple binary classifiers at the same time and sharing features among them. The algorithm has the advantage that less training data is needed since many classes can share similar features. Readers are invited to consult 9] for details about the algorithm. In contrast to 9, in our model, combined features are shared among the classes instead of sharing a single feature. This is done through the weak learner used by our learning model (presented in 7]) which is different from the one used in 9 .

\section{Experimental Evaluations}

The presented GOR model is evaluated experimentally to analyze its benefits and limitations. The performance is measured in three cases. First, using only appearance information for recognition. Second, using only 3D (range) information. Finally, using a combination of both types of information. The model, for each previously mentioned case, is trained in two ways: 1) independently, 2 ) jointly with feature sharing among classes. For all experiments, the number of training iterations (number of weak hypotheses) is fixed to $T=150$ and is independent of the number of classes. In contrast to the learning model in 9], we are not searching and comparing the learning effort for a certain error rate but we report the ROC-equal-error rate for a certain learning effort, namely $T$ weak hypotheses. All experiments are performed using our new object category dataset. We use five classes: cars, fruits, animals, toys and cups (see figure 31). The number of training examples for each class is 100 examples which results in a total of 500 training examples. For testing, 60 examples per class (images of new instances) are used (a total of 300 examples).

Recognition using appearance information only: The aim of this set of experiments is to measure the performance of the model using only appearance-based 
Table 1. Comparison of the ROC-equal-error rates of the appearance (2D) and range based descriptors used separately and combined for learning and classifying the five object classes (with independent learning)

\begin{tabular}{lrrrrrc}
\hline Descriptors & \multicolumn{3}{c}{ Cars Fruits Animals Toys Cups $\begin{array}{c}\text { Avg. error } \\
\text { over classes }\end{array}$} \\
\hline Appearance-Desp. & 0.300 & 0.200 & 0.250 & 0.283 & 0.233 & 0.253 \\
Range-Desp. & 0.417 & 0.500 & 0.317 & 0.183 & 0.363 & 0.356 \\
\hline Appearance + range Comb. & 0.370 & 0.167 & 0.200 & 0.183 & 0.183 & 0.221 \\
\hline
\end{tabular}

Table 2. Confusion matrices of multi-class recognition (with independent learning) results using appearance (2D) descriptors, range descriptors and the appearance-range combination respectively. For the computation of the confusion matrix, the best classification of an image over all classes is counted as the object category. Numbers represent percentage $(\%)$ of test images (60 images per class) classified for each class. Columns represent true classes.

\begin{tabular}{l|ccccc|cccccc|cccc}
\hline & \multicolumn{4}{|c|}{ Appearance Desp. } & \multicolumn{4}{|c|}{ Range Desp. } & \multicolumn{4}{|c}{$\begin{array}{c}\text { App.+Range } \\
\text { Comb. }\end{array}$} \\
\hline Class & c1 & c2 & c3 & c4 & c5 & c1 & c2 & c3 & c4 & c5 & c1 & c2 & c3 & c4 & c5 \\
\hline Cars:c1 & $\mathbf{4 7}$ & 1 & 3 & 8 & 3 & $\mathbf{7 0}$ & $\mathbf{8 2}$ & 32 & 25 & $\mathbf{6 5}$ & $\mathbf{5 3}$ & 5 & 5 & 5 & 7 \\
Fruits:c2 & 13 & $\mathbf{7 7}$ & 12 & 1 & 12 & 2 & 17 & 1 & 8 & 2 & 15 & $\mathbf{8 0}$ & 8 & 5 & 3 \\
Animal:c3 & 15 & 2 & 33 & 1 & 5 & 18 & 1 & $\mathbf{3 7}$ & 5 & 18 & 3 & 0 & $\mathbf{3 2}$ & 0 & 7 \\
Toys:c4 & 7 & 12 & 13 & $\mathbf{5 3}$ & 3 & 5 & 0 & 11 & $\mathbf{6 0}$ & 0 & 12 & 5 & 23 & $\mathbf{7 8}$ & 5 \\
Cups:c5 & 18 & 8 & $\mathbf{3 8}$ & 27 & $\mathbf{7 7}$ & 5 & 0 & 10 & 2 & 15 & 17 & 10 & $\mathbf{3 2}$ & 12 & $\mathbf{7 8}$ \\
\hline
\end{tabular}

information. A combination of the SIFT and color descriptors is used for learning and recognition. Learning the five classes is performed independently. The recognition performance (ROC-equal-error rates) using the test images is displayed in table 1. It should be noted that in our learning model, the background class for learning and classifying one object class (or a subset of classes) is a combination of the other classes. Multi-class learning and classification in such a case is a difficult task as the background class is very heterogeneous in appearance and is much more likely to appear than the various object classes since most of the image is background. This affects in turn the final recognition performance of the model.

Recognition using range information only: A combination of the local shape descriptors (range-based information) is used here alone for learning and recognition. Table 1 displays the recognition performance. Generally, the recognition performance using range-based information is lower than the performance using the appearance-based information. This could be argued to the low resolution of the intensity images of the TOF camera, which are used for point detection when range images are used for recognition. This low resolution of the images (which are, additionally, full of background clutter) affects the detection performance of the point detector and influences, in turn, the classification 
Table 3. Comparison of the ROC-equal-error rates of the appearance (2D) and range based descriptors used separately and combined for learning and classifying the five object classes (with joint learning)

\begin{tabular}{|c|c|c|c|c|}
\hline Descriptor & Cars Fruits & Animals & Toys Cups & $\begin{array}{l}\text { Avg. error } \\
\text { over classes }\end{array}$ \\
\hline Appearance-Desp. & $0.267 \quad 0.250$ & 0.220 & 0.3500 .317 & 0.280 \\
\hline Range-Desp. & $0.400 \quad 0.550$ & 0.440 & 0.2600 .350 & 0.400 \\
\hline Appearance + range Com. & 0.3830 .300 & 0.280 & 0.2170 .250 & 0.306 \\
\hline
\end{tabular}

performance. Moreover, the noisy nature of the TOF range images affects the construction of a clear shape representation for each class which has an effect on the recognition performance.

Recognition using a combination of appearance and range based information: To assess the performance of the model when using different types of information (appearance and range), a combination of the appearance and range based information is used for training and testing the recognition model. Again, learning is performed independently. The recognition performance is shown in table 11. The combination of the three different types of descriptors improves the performance over almost all classes. The performance gain using the appearance-range combination is $35 \%$ for the best single class (toys) and $12 \%$ over classes, which reveals the benefits of combining both different types of information for recognition. Table 2 shows the confusion matrices of recognition using appearance-based information, range-based information and appearancerange combination respectively. The confusion using only range information is high in comparison to the case of appearance information, while the confusion is improved by using appearance-range combination.

Joint vs. Independent Learning: To assess the performance of joint learning, the experiments are repeated with the classes being learnt jointly with feature sharing. Table 3 displays the results of joint learning. It can be noted that the joint learning (table 3) is not suitable for our recognition case as it does not significantly achieve better performance than the independent learning (table 1). The recognition performance using idependent learning is better (achieves lower error rates) in most of the cases than joint learning.

\section{Conclusions}

This paper has presented two contributions. First, a new object category dataset has been constructed. The dataset has the advantage over existing datasets that it provides both $2 \mathrm{D}$ and range data of its member classes and can be used for both 2D and 3D generic object recognition (GOR). Second, a GOR model for multi-classification of visual object classes from range images using shape information has been proposed. Also, the general framework of the model allows 
the use of appearance-based information extracted from 2D images for recognition. Moreover, it is able to exploit a combination of both appearance-based (extracted from 2D images) and shape-based (extracted from range images) information for recognition of multiple object classes. Experimental evaluation of the model using the two different types of information has shown good performance. However, combining the two different information types improves the recognition performance.

\section{References}

[1] Fei-Fei, L., Fergus, R., Perona, P.: Learning generative visual models from few training examples: An incremental bayesian approach tested on 101 object categories. In: 2004 Conference on Computer Vision and Pattern Recognition Workshop, p. 178 (2004)

[2] Fergus, R., Perona, P., Zisserman, A.: Object Class Recognition by Unsupervised Scale-Invariant Learning. In: IEEE Computer Society Conference on computer vision and Pattern Recognition CVPR3, June 2003, vol. 2, pp. 264-271 (2003)

[3] Hetzel, G., Leibe, B., Levi, P., Schiele, B.: 3d object recognition from range images using local feature histograms. In: IEEE International Conference on Computer Vision and Pattern Recognition (CVPR 2001), vol. 2, pp. 394-399 (2001)

[4] Lange, R.: 3D Time-of-Flight Distance Measurement with Custom Solid-State Image Sensors in CMOS/CCD-Technology, PhD thesis, University of Siegen (2000)

[5] Lowe, D.G.: Distinctive image features from scale-invariant keypoints. International Journal of Computer Vision 60, 91-110 (2004)

[6] Mikolajczyk, K., Schmid, C.: An affine invariant interest point detector. In: Heyden, A., Sparr, G., Nielsen, M., Johansen, P. (eds.) ECCV 2002. LNCS, vol. 2350, pp. 128-142. Springer, Heidelberg (2002)

[7] Opelt, A., Pinz, A.: Object localization with boosting and weak supervision for generic object recognition. In: Kalviainen, H., Parkkinen, J., Kaarna, A. (eds.) SCIA 2005. LNCS, vol. 3540, pp. 862-871. Springer, Heidelberg (2005)

[8] Ruiz-correa, S., Shapiro, L.G., Meil, M.: A new paradigm for recognizing 3-d object shapes from range data. In: Proceedings of the IEEE Computer Society International Conference on Computer Vision 2003, vol. 2, pp. 1126-1133 (2003)

[9] Torralba, A., Murphy, K.P., Freeman, W.T.: Sharing visual features for multiclass and multiview object detection. IEEE Transactions on Pattern Analysis and Machine Intelligence 29(5), 854-869 (2007)

[10] van de Weijer, J., Schmid, C.: Coloring local feature extraction. In: Leonardis, A., Bischof, H., Pinz, A. (eds.) ECCV 2006. LNCS, vol. 3952, pp. 334-348. Springer, Heidelberg (2006) 